heure et pendant ce temps on chauffe le liquide antiseptique cl les traverses qu'il bargne à l'aide de serpentins placés à l'intérieur du cylindre. On rétablit la pression atmosphérique dans le cylindre, ce qui permel au liquide antiseptique de regagner celui-ci par l'effet de la différence de pression exislant entre le 'ylindre et le réservoir. L'air contenu dans chaque cellule se détend alor's, expulse la créosote et ne laisse de cette dernière que la quantité nécessaire pour imprégner les parois des cellules.

'our cortames essences, le chêne et lo pin en particulier, celle oricilon suffit. Pour le hètre, l'imprégnation complèle crige deux fois le même cycle d'opérations.

La nécressité de soumettre à un double cycle d'opérations d'injeclion al de pression les traverses de hêtre provient de la minceur des parois des cellules ef des vaissean de ec bois. lors do la première compression, lo liquide antiseptique no prul pénélrer suffisamment, l'intéricur du bois n'étant pas à ine tempéralure suffisante et la naphtaline contenue dans la créosote obstruant los orifices capullaires on s'y déposant Prudant le second cycle, le bois étant mieux chauffé, Is liquidr reste plus fluide, c'est-à-dire moins pâteux, et pénètro lo bois aussi loin que cola est nécessairc pour sa conservation.

Au point de vur de l'économie róalisée, le procédé Rüping présente un avantage sérieux sul les méthodes ordinaires. Fr offel, alors que dans le procídé ordinaire par vide ol injection sous pression, la quantité de créosole absorbé varie entre 6 et 8 kilogrammes par lraverse de chêne et cntro 76 ol 30 kilogrammes par traverse de hêtre, olle n'est que de $\{$ à 5 kilogrammes pour lo chêne cl de is à r 6 pour le hêtre par l'application du procédé Riiping. En outre, la manipulation des pièces de bois est plus facile, la résistance mécanique de celui-ci est augmentée d'une façon très appréciable à toutes les essences.

Procédé Gefateau et Merklen. - Il nous reste à dire quelques mots du procédé Château cl Merklen basé sur ce fait que l'injection des traverses trop sèches se fait dans de mulaises conditions. Pour ob’vier à cel inconvénient, le bois est sounis, dans une première opération, à l'action alternative de la rapeur et du vide. la vapeur apporte l'eau nécessaire à l'humidification des régions trop sèches, chauffe en même temps le bois dans toute sa masse et évitè ainsi la solidification possible de la créosnte pendant l'injection ; en outre, elle stérilise et dilue la sève. I,e vide vaporise l'ean condensée, extrait les gaz et une partic de la sève contenus dans le bois en l'empêchant ainsi de se décomposer.

Une fors introduite dans le cylindre, la créosote est injecléc dans le boiśà l'aide d'une pompe qui élève la pression juaqu'à 8 atmosphères.

Dans cette deuxième opération, les traverses absorbent loule la quantité de créosote nécessaire à leur imprógnation romplète. Cette dernière ćlant terminée, l'excès de créosote -écoule en dehors du cylindre el on introduil à sa place dr l' 1 apcur sous pression. Ia température doit alteindre cnviron $135^{\circ}$ dans le cylindre ; on y introduil alors de l'air comprimé à la température ordinaire. En se condensant, la vapcur abandonne ses calories à la créosolo contenuc dan le bois et la maintient ainsi bien fluide ; l'air comprimé joue le rôle de piston sur cette dernière et la rofoulc encore phus en avant dans l'intérieur du bois. On termine l'opération par une nouvelle compression de vapeur pendant laquelle la température atteint progressivenient 150 degrés.
(A suivre.)
J. EscírD, Ingénieur civil, Lauréat de l'Institut.

\title{
REVUĚ DES SOCIÉTÉS SAVANTES ET DES PUBLICATIONS SCIENTIFIQUES
}

\section{ACADÉMIE DES SCIENCES}

\section{ÉLECTRICITÉ}

Sur l'influence de l'envoloppe sur les résistance et réactance effectives d'un' cible armé pour les harmoniques 3. - Note ( ${ }^{1}$ ) de M. Swyngedauw, transmise par M. Blondel.

Un trouçon d'environ $16 \mathrm{~m}$. de câble $3 \times 100 \mathrm{~mm}^{2}$ de même spécification que les câbles en service étudiés précédemmént, a été coupé en deıx fragments ayant chacun $8 \mathrm{~m}$. de long environ, plićs en $U$ dont les branches parallèles étaient espacées d'environ $8_{0} \mathrm{~cm}$.

L'un des tronçons est d'abord dépouillé de son feuillard, l'autré conservé intact.

On mesnre, pour chacun d'eux, le courant, la tension, la puis sancé absorbés et l'on déduit de ces données les résistance et réac tance kilométriques comme dans les essais précédents.

Ces premiers essais donnent par comparaison l'influence $\mathrm{du}$ feuillard.

On débarrasse ensuite, de son enveloppe en plomb, le tronçon déjà dépourvu de son feuillard.

De nouvelles mesures donnent l'influence de l'enveloppe.

Principaux résultats :

Cable nu, sans enveloppe, ni feuillard. - A la fréquence 50

(1) Séance du 6 janvier 1919. périodes, lá résistance est égrale à $\mathrm{r}, \mathrm{I}$ fois la résistance en courant continu ; à la fréquence 500 , elle égale neuf fois cette dernière.

La réactance est proportionnelle à la fréquence.

Influence de l'enveloppe. - Le câble recouvert ou non de son enveloppe garde sensiblement la même résistance aux fréquences expérimentées ( 50 à 500 péríodes pảr seconde).

La réactance est nettement diminuée par l'enveloppe. Pour $\mathrm{F}=500$ périodes la réactance du tronçon muni de son enveloppe est les trois quarts de celle du câble nu.

Influence de l'armature: - A la fréquence 5o périodes, la résistance et la réactance commencent par croître avec le courant jusqu'à $i=70$ ampères qui correspond à un champ inducteur $\mathscr{H}=4$ gauss dans le feuillard. Flles restent ensuite constantes jusqu' $\mathrm{i}=\mathrm{x} 20$ ampères correspondant à un champ $\mathcal{H}=7$ gauss. Elles diminuent ensuite lentement et régulièrement quand le courant continue de crôitre.

La résistance ét la réactance kilométriques sont deux à trois fois plus grandes pour les tronçons courts que pour les longs câbles en service.

A la fréquence 500 périodes, ces grandeurs atteignent jusquà quatre fois les valeurs correspondantes des longs câbles, de sorte qu'il est impossible de déterminer à l'usine, sur des tronģons de quelques mètres, les résistances ét réactances des cables en service, pour l'harmonique 3.

Cette énorme différcnce entre les valeurs des constantès, pour Ics câbles longs et courts, semble due à l'enveloppe.

Entre les résistance et réactance kilométriques de deux lóngs 
càbles de même spécification posés dans la mệme tranchée ou séparés de $I \mathrm{~km}$. dans leur parcours, on observe une diftérence de l'ordre de 20 lois plus farble que la valeur calculée pour les mèmes câbles dépoullés de leurs enveloppe et feuilıard : résultat qui s'explique assément par le tlux antagoniste créé par le courant induit dans l'enveloppe.

Cetle explıcalion est corroborée par ce fait que l'énergie dégagée dans les feuillards est considérablemeut mons élevée que celle qui correspondrait au champ qu'y créerait le courant enculant dans les âmes s'il agissait seul.

Le courant indul dans l'enveloppe d'un càble posé en terrain parfaitement isolant serait uaiquement un courant de capacite el, comme cetle capacité dépend de la longueur du càble, on conçonl déjà que le courant induit dans lenveloppe peul etre d'autant plus intense que le câble est plus long ; la relation que l'on peul établir dans cette hypothèse rend comple de l'inlluence de la longueur sur les constantes spécifiques.

Lin réalité, comme le terrain où le câble est posé n'est pas un ssolant parfait, des courants de conduction sajuutent aux précédents el les conslantes en sont notablement modilées.

Les resistance el réactance effectives linéiques pour les harmonques 3 doivent ètre détermnées sur les cables en service ; clles ne sont pas des constantes déterminées uniquement par la specilcation du câble comme pour les autres harmoniques.

\section{UNIVERSIDAD' NACIONAL DE LA PLATA}

rublications de la raculte des berences (avil 1919, $0^{\circ} 42$ ).

\section{Choix du type d'ouvrage pour une prise de dérivation.}

Le choix du lype d'ouviage à adopter pour la construction dunc prise est une question exclusivement connee au wons sens perounel de l'ingénicur. Heme lorsqu'll y a possibilite de lane intervenir dans une etude théorique les muliples cricoustances locales qui, dans chaque cas particulier devront etre prises en considération au moment de choisir le type d'ouvrage, on a cher ché, dans la présenle publication, à déduire quelques iègles génerales dérıvées de l'étude de la manière dont s'effectue le tramage de lond dans les rivières ou tleuves de pente rapide.

Liffet immédiat d'une prise fixe est celui d'opposer un obstacle eflicace à l'arrèt des matériaux charriés occasionnant ainsi au lond de la rivière un banc en amont de la prise.

Il est possible de prévoir l'inlensité et l'effet du banc que produira un ouvrage lixe, par l'étude des lois qui gouvernent le trainage de lond, et la première partıe de ce travail s'occupe d'établir ces lois.

L'étude'du trainage de fond qui se prête à de nombreuses applicalions pour lout ce qui se rapporte à la correction de torrents, lit de rivières, etc., permet d'obtenir facilement l'expression $J=\frac{z_{0}{ }^{3} i}{(z-n)^{3}}$ qui donne la relation entre la pente superficielle $J$ el la profondeur $(z-\eta)$ en un point quelconque en fonction de la prolondeur $z_{0}$ el de la pente $i$ originaires du cours d'eau avant d'établir la prise. Cette équation que l'on pourrait aussi utiliser your la détermination de la courbe d'eau dormante produite par une digue, en tenant comple de la surélévation du fond due aux bancs, unie aux équations qui déterminent le volume $b$ des malérlaux emporté permet de connaître la profondeur $h_{1}$ de l'eau, immédiatement en amont de-la prise, et par conséquent de détermiler s'il se produirá ou non une surélévation du fond, nuisible au hon fonctionnement des vannes de la prise. Comme conclusion, on arrive à établir que dans les rivières dont la pente est supérieure $\frac{1}{222}=0,0045$, il est à craindre que le lit s'obstrue com plètement par des banas en amont de la digue de prise, el par conséquent, il convient dans ces cas, de recourir à l'emploi de prises mobiles ou demi-fixes.

\section{Méthode pour varier l'angle de phase d'un courant en maintenant constante son intensité et sa tension.}

En étudiant les phénomènes des circuits as ec aulo-induction, capacité et résistance, il se présente le cas surant qui n’a pas cncore été trasté, selon l'auteur.

En connectant une aulo-induction et une résstiance en série, Flaçant une capacilé en dérivation sur le crrcuil el lassant varier la résistance, on conslate que l'intensité totale du circuit varie de telle manière que le lieu géométrique de son diagramme vectoriel est un cercle dont le centre se trouve sur la ligne normale au vecteur de la tension constante. Si, en plus, on choisit la capacité et l'auto-induction de telle manière que

$$
\frac{1}{2 \pi \nu \mathrm{L}}=4 \pi v \mathrm{C}
$$

il résulte que l'intensilé esl indépendante de la résistance donl la valeur seule détermine le facteur de puissance à intensité cons. lante.

\section{REVUE DES PUBLICATIONS ETRANGËES}

\section{CHEMICAL NEWS}

Vulcanisation du GaOUTCHOUC PAR LE SÉLÉNIUM

C.-R. Boggs dans Industrial Eng. Chem. J. (10-2, p. 117-118, fév. rg18) et également dans Chem. lews (11-, p. I99-200, $24 \mathrm{ma}$ 1918 ) cite les expériences fartes en vue de valcaniser le caoutchou avec le sélénım. La variété nore cristallıne du sélénium londant à $2 I 7^{\circ}$ est utilisée, la variété amorphe se transforme en celle-cı entre 100 el $r 50^{\circ}$. On utilise une quantité de sélénium (variété cristalline et amorphe) équivalente à celle du soufre utilisé ordiuairement; en chaulfant deux heures à I $50^{\circ}$ un produit vulcanisé est obtenu, capable de rester en bon état duranl quatre ans. Liependaut il n'a pas la résiślance du caoutchouc vullcanisé ordinaire. Ln chaulfage plus prolongé n'améliore pas le résultat, si l'on emploie deux fors plus de sélénium le caoulchouc se délériore. En utilssant des accúlérateurs organiques que l'auteur ne désigne pas, el en chauffant à $135^{\circ}$ pendant un temps double, de crlui nécessarre dans l'opération au soufre, des caoulchoucs de force el d'allongement normaux sont obtenus; leur valeur dielertrique est inférieure à celle du caoutchouc vulcanisé ordinaire, mais ils so conservent bien. Luanalyse de ces produits est asséz difficile, te sélénium étant difficlement soluble dans les dissolvants ordinaires du caoutchouc: acétone, chloroforme el sulfure de carbone. Quelques points assez curieux de celte préparation se trouvent dans le fall que la vulcanisalion se produit à une température inférıeure au point de fusion du sélénium et que le caoutchouc sélénié ne se délériore pas, malgré la sensibilité du séléniữm à la lumière.

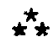 \\ ELEMTRISCHES ZEITSCHRIFT}

SYSTEME DE IRANSUISSION ÉLECTRLQUE POUR BESOINS AGRICOLES (H. Rolh. Elelit. Zeits, pp. ì 3, I $^{\text {er }}$ Mars 1918).

bans une transmission à grande distance, des réseaux formés de système de haut, moyen ct bas voltage sonl utilisés; le vollage moyen peul ètre régularisé dans les sous-stations par des régulateurs de voltage. Comne les voltages élevés ne peuvent pas toujours être appliqués directement à ces régulateurs, la méthode employée ordinairement consiste à suppléer les, régulateurs du système à voltage moyen par des transformateurs de courant. Lnc amélioration de cette méthode consiste à transformer le haut en bas voltage par un transformateur étoilé ; le régulateur élant en connexion avec le transformateur principal et son point neutre. En appliquant le système aux besoins agricoles, des calculs seront faits pour détcrminer l'aire que chaque sous-station ṕcut nourrix. La difficulté spéciale dans l'établissement d'un réseau pour les besoins agricoles se trouve dans ce fait que le système est plutòt un réseau distributeur qu'un réseau de transmission, cela rend la 